\title{
ADMINISTRACIÓN EN EMPRESAS QUÍMICAS DE MEDELLÍN CON TENDENCIA \\ A LA ORGANIZACIÓN 4.0*
}

\section{ADMINISTRATION OF CHEMICAL COMPANIES IN MEDELLIN WITH A TENDENCY TOWARDS THE ORGANIZATION 4.0}

Recibido: 4 de septiembre de 2019

Evaluado: 8 de octubre de 2019

Aprobado: 10 de diciembre de 2019

\author{
Juan Santiago Calle Piedrahita ${ }^{* \star}$ \\ Universidad Nacional de Colombia \\ Orcid: https://orcid.org/0000-0001-6320-5204 \\ Gloria María Isaza Zapata** \\ Universidad de Almería \\ Orcid: http://orcid.org/0000-0002-7699-8714
}

Ana Isabel Isaza Zapata ${ }^{* * * *}$

Universidad de Maimodenis

Byron Enrique Portilla Rosero ${ }^{\star * * * \star}$

Universidad Nacional de Colombia

Cómo citar este artículo: Calle Piedrahita, J. S., Isaza Zapata, G. M., Isaza Zapata, A. I., y Portilla Rosero, B. E. (2020). Administración en empresas químicas de Medellín con tendencia a la organización 4.0. Revista Estrategia Organizacional, 9(1). doi: https://doi. org/10.22490/25392786.3641

* $\quad$ Artículo de investigación.

** Doctor en ingeniería - Universidad Nacional de Colombia. Administrador de negocios, especialista en mercadeo, Magíster en Administración. Docente - investigador del grupo Goras - Universidad católica Luis Amigó. https://orcid.org/0000-0001-6320-5204 juan.callepi@amigo.edu.co

*** Candidata a doctora en Educación. Universidad de Almería. Grupo de investigación Pedagogías y didácticas de los saberes. Docente Universidad Pontificia Bolivariana. Correo electrónico: gloria.misaza@gmail.com

$\star \star \star \star$ Doctora en Psicología. Universidad Maimonedis. Docente Universidad Católica Luis Amigo. Correo electrónico anais@une.net.co

$\star \star \star \star \star$ Magister en ingeniería de sistemas. Universidad Nacional de Colombia. Correo electrónico. byronpor@gmail.com 


\section{RESUMEN}

Introducción: Las empresas de químicos tienen un enfoque hacia las ventas y no hacia el mercadeo lo cual ha sido motivo que dificulta para incorporarse en una organización 4.0 (Li, 2018). El objetivo del artículo es identificar las características de las empresas de químicos para lograr adaptarse a las organizaciones 4,0 desde una visión del mercadeo. Las organizaciones 4.0 son un modelo de negocio donde participan los comportamientos de los clientes digitales, lo cual fuerza a una modificación de la gestión administrativa tradicional del mercadeo. Metodología: es cuantitativa, se desarrolla mediante un cuestionario con 19 preguntas cerradas y dos abiertas realizada durante los años 2017- 2018, donde participaron 16 directivos de empresas químicas en Medellín. Analizadas con el programa de prospectiva MICMAC. Resultados: Los principales hallazgos se orientan a la certificación, logística y gestión de las relaciones del consumidos (CMR). Es decir, las organizaciones 4.0 desde la química tienen un compromiso de relacionar lo interno con lo externo. Las empresas de químicos deben buscar la gestión de la innovación, la administración de los datos que se convierta en información en tiempo real; es decir, incorporar conocimiento táctico y explícito desde una movilidad interna y externa de la organización con una visión inter-operatividad. Conclusiones: las empresas químicas para empezar una organización 4.0 hacia el 2030 , ligadas al desarrollo sostenible deben comprender a sus clientes como si fuesen parte interna de la compañía, además de establecer acciones de transformación ancladas en la actualización. Las implicaciones a futuro de las empresas químicas es relacionar la tecnología y la innovación con una propuesta ambiental.

Palabras clave: gestión, organización, tecnología, comercio, innovación científica, administración.

\section{ABSTRACT}

Chemical companies have a focus on sales and not marketing, which has been a reason that makes it difficult to join a 4.0 organization ( $\mathrm{Li}, 2018$ ). The objective of the article is to identify the characteristics of chemical companies in order to adapt to organizations 4.0 from a marketing perspective. Organizations 4.0 are a business model where the behaviors of digital customers participate, which forces a traditional management modification of the traditional marketing administration. The methodology is quantitative; it is develop through a questionnaire with 19 closed and 2 open questions made during the years 2017-2018, where 16 managers of chemical companies in Medellín participated. Analyzed with the MICMAC prospective program. The main findings are oriented to the certification, logistics and consumer management relationships (CMR). 
That is, 4.0 organizations from chemistry have a commitment to relate the internal to the external. Chemical companies must seek innovation management, data management that becomes information in real time; that is to say, to incorporate tactical and explicit knowledge from an internal and external mobility of the organization with an inter-operability vision. In conclusion, chemical companies to start a 4.0 organization by 2030 , linked to sustainable development must understand their customers as if they were an internal part of the company, in addition to establishing transformation actions anchored in the update. The future implications of chemical companies is to relate technology and innovation with an environmental proposal.

Keywords: Management, Organization, Technology, Commerce, Scientific Innovation, Administration.

\section{INTRODUCCIÓN}

Las empresas de químicos en Medellín, en su mayoría, centran su interés en la orientación a las ventas, pocas hacia el mercadeo y una organización 2.0. Esto configura un elemento complejo, cuando se analizan las compras, la comercialización por otros medios; los cuales, actualmente se basan en técnicas de asesoramiento personal y de despacho "face to face".

Por consiguiente, las empresas tienen una mirada de compras vinculando nuevas tecnologías de tipo online y el uso de dispositivos inteligentes nuevos. Además, generan cargos expeditos a nivel laboral al consolidarse, entre otros, jefes de compras que revisan por internet nuevas cotizaciones y toman decisiones en relación a los precios, ubicación, logística, nuevas tecnologías, seguridad informática y servicio.

Ahora bien, las empresas químicas se consideran que son organizaciones contaminantes; sin embargo, desde el enfoque de los objetivos de desarrollo sostenible de la ONU en su objetivo nueve (industria, innovación e infraestructura) en el literal 9.2 el cual consta de "promover una industrialización inclusiva, sostenible y, de aquí a 2030, aumentar significativamente la contribución de la industria al empleo, el producto interno bruto" (ONU, 2015). En consecuencia, las organizaciones 4,0 de productoras de químicos en Medellín se apoyan en la gestión de innovar en la industrialización inclusiva, pero con apoyo de un mercadeo actualizado e innovador. 
Esto conlleva a preguntar ¿cuáles son las características de mercadeo y ventas que deben de tener las empresas de químicos en Medellín a enfocadas a las organizaciones 4,0 para el año 2030? El objetivo del artículo es identificar las características de las empresas de químicos para lograr adaptarse a las organizaciones 4,0 desde una visión del mercadeo y el desarrollo sostenible.

La industria 4,0 vistas desde investigaciones alemanas de Lasi, Fettke, Kemper, Feld, y Hoffmann (2014) se centran en mejorar el uso de la tecnología de información y automatización de las fábricas (Kagermann, Helbig, Hellinger, y Wahlster, 2013). Es decir, las organizaciones con orientación al mercadeo se apoyan en la tecnología de la información, de la comunicación para mejorar los procesos de contacto con el cliente desde la gestión las relaciones con el consumidor (CRM). Además, al incluir Cyber-Physical-Systems, la comunicación avanzada entre máquinas equivale a su diálogo con los humanos (Brettel, Friederichsen, Keller, y Rosenberg, 2014) como un medio nuevo para impulsar el mercadeo internacional con una visión local. Esto en sí, transforma la gerencia hacia un desarrollo sostenible químicamente.

\section{ORGANIZACIONES 4.0}

Las diversas investigaciones apuntan a identificar las organizaciones 4.0 enfocadas a la tecnología e innovación. Por tanto, el escrito de Galletta, Carnevale, Celesti, Fazio e Illari (2017) expone que as estrategias de marketing son formas de "retención" dirigidas no solo a la adquisición de nuevos clientes sino también a la rentabilidad de los existentes. Además, Henning, HagedornHansen y Von Leipzig (2018) expusieron que la industria 4.0 deben analizar los datos en tiempo real con tecnología y no en forma manual (Han, Lee y Choi, 2013; Nadarajah y Kotz, 2008; Chikez, 2016; Mayer y Nusswald, 2001) (ver tabla 1).

Tabla 1. Investigaciones de Organizaciones 4.0

\begin{tabular}{|l|l|l|l|}
\hline \multicolumn{1}{|c|}{ Autor } & \multicolumn{1}{|c|}{ Tema } & \multicolumn{1}{c|}{ Metodología } \\
\hline $\begin{array}{l}\text { Ardito, Petruzzelli, } \\
\text { Panniello y Garavelli, } \\
(2019) .\end{array}$ & $\begin{array}{l}\text { Industria 4.0 y } \\
\text { los sistemas de } \\
\text { información }\end{array}$ & $\begin{array}{l}\text { Análisis de ejemplos reales } \\
\text { (casos) }\end{array}$ & $\begin{array}{l}\text { Tecnologías habilitadoras para la } \\
\text { Industria 4.0 que pueden servir } \\
\text { particularmente para administrar la } \\
\text { interfaz SCM-M desde una perspectiva } \\
\text { de procesamiento de información. }\end{array}$ \\
\hline $\begin{array}{l}\text { Fremont, Frick, Åge } \\
\text { y Osarenkhoe (2019). }\end{array}$ & $\begin{array}{l}\text { Controversia y } \\
\text { fricción dentro de } \\
\text { la digitalización }\end{array}$ & $\begin{array}{l}\text { Empírico-analítico dos } \\
\text { organizaciones que están tratando } \\
\text { de mejorar su ventaja competitiva } \\
\text { a través de la digitalización }\end{array}$ & $\begin{array}{l}\text { Los datos en la industria 4.0 son } \\
\text { modularidad, estandarización, } \\
\text { abstracción y tangibilidad. }\end{array}$ \\
\hline
\end{tabular}




\begin{tabular}{|l|l|l|l|}
\hline \multicolumn{1}{|c|}{ Autor } & \multicolumn{1}{|c|}{ Tema } & \multicolumn{1}{c|}{ Metodología } \\
\hline $\begin{array}{l}\text { Gornostaeva, Lazareva, } \\
\text { Bugaeva, Gribova } \\
\text { y Zibrova (2018). }\end{array}$ & $\begin{array}{l}\text { Emprendimiento en } \\
\text { industria 4.0 }\end{array}$ & Análisis estadístico & $\begin{array}{l}\text { La industria 4.0 es baja por la falta } \\
\text { de incentivos para el mercado }\end{array}$ \\
\hline $\begin{array}{l}\text { Karaosman, } \\
\text { Morales-Alonso } \\
\text { y Grijalvo (2015). }\end{array}$ & $\begin{array}{l}\text { Consumidores a la } \\
\text { RSE en un entorno } \\
\text { intercultural desde } \\
\text { la industria 4.0 }\end{array}$ & $\begin{array}{l}\text { Investigación cualitativa } \\
\text { exploratoria para la cual } \\
\text { entrevistas grupales, incluyendo } \\
\text { seis discusiones grupales con } \\
\text { consumidores españoles y turcos }\end{array}$ & $\begin{array}{l}\text { Perciben las acciones de RSE } \\
\text { como parte de las estrategias de } \\
\text { marketing de las empresas, mientras } \\
\text { que la conciencia general de los } \\
\text { consumidores sobre la RSE es baja. }\end{array}$ \\
\hline
\end{tabular}

Fuente: elaboración propia

Las organizaciones 4.0 hacia futuro se estructuran en comprender la tecnología, innovación, sistemas, minería de datos y digitalización de los datos.

\section{MERCADEO DE ORGANIZACIONES 4.0}

El mercadeo de las organizaciones 4,0 según expone Roblek, Meško, y Krapež (2016) se basa en tecnologías conectadas a internet para la creación de valor agregado. Además, la innovación en el mercado laboral y de mercadeo es un elemento diferenciador en el siglo XXI (Palazzeschi, Bucci, y Di Fabio, 2018); las organizaciones 4,0 se deben enfocar a involucrar innovación y utilizar el internet como elemento para efectuar negociaciones globales y no solo locales.

Igualmente, Ferrare (2019) enuncia que urgen desafíos de seguridad con cada nueva tecnología utilizada, pero se pueden evitar robos o ataques a futuro al tener constamente actualizaciones de los sitemas de compras y mercadeo institucional. Del mismo modo, la seguridad es un mecanismo de defensa de los protocolos que aportan a mejorar la implementación de las organizaciones 4,0 (Alani y Alloghani, 2019). Pero, Wang et al., (2019), presenta que es necesario gerenciar la transcripción de datos para mediar la reprogramación, y evitar la penetración del sistema al realizar una venta por medios no tradicionales. Desde este enfoque las organizaciones 4,0 basadas en el mercadeo online deben efectuar cosntamente revisiones de los sistemas de seguridad para comercializar mejor los productos y evitar robo de entidad.

A su vez, aporta a tener una información detallada de los clientes para mejorar las ventas y el servicio intrínseco no personalizado. Pero Shipton, West, Parkes, Dawson, y Patterson, (2006) identificaron que la satisfacción laboral agregada es fundamental para ejercer una innovación organizacional que aporta a mejorar la rentabilidad y a su vez impulsa una gestión de mercadeo sostenible. 
Al mismo tiempo, las redes inteligentes en el uso de las TIC configuran la generación de cadena de valor en las organizaciones con una visión del 4,0 (Mrugalska y Wyrwicka, 2017). Las organizaciones 4,0 se actualizan en la gestión de sus procesos internos a partir de: mecanismo inteligente, protocolos de seguridad autónomos, gerenciar el big data, establecer modelos de análisis de los sistemas de modulaciones dinámicas, identificar la seguridad cibernética, procesar las maquinas físicas con tecnología modular e identificar problemas de inversión en tecnología e innovación (Vaidya, Ambad, y Bhosle, 2018).

Pero, no es solo tener tecnología avanzada, se debe analizar e incorporar gestión en la cadena de suminsitro, tranformación digital y cooperación inteligente para estar cerca de los clientes y sus necesidades (Barreto, Amaral, y Pereira, 2017). Así mismo "las organizaciones 4,0 cambiaran significativamente los productos, sistemas de producción en diseños de procesos y operaciones" (Pereira y Romero, 2017, p. 1212). Las organizaciones 4,0 se apoyan en la tecnología e innovación como elemento que aporta a realizar reingeniería en ellas para comprender al cliente interno y externo, para así mejorar las operaciones. Igualmente, se identificó el mecanismo de interrelación entre factores de innovación y la tecnología (Sony y Naik, 2019): calidad, producto, servicio, gestión, compromiso de los directivos y mercadeo

\section{METODOLOGÍA}

La investigación realizada se orientó desde el paradigma cuantitativo, el cual permite considerar desde un estudio descriptivo como lo enuncia Hernández, Fernández y Baptista (2010) "busca especificar las propiedades, las características y los perfiles de personas, grupos, comunidades, procesos, objetos o cualquier otro fenómeno que se somete a un análisis. (p.80).

Tabla 2. Definición de variables en el estudio

\begin{tabular}{|l|l|l|}
\hline No. & \multicolumn{1}{|c|}{ Variable } & \multicolumn{1}{c|}{ Explicación } \\
\hline 1 & Certificación de Calidad & ISO 9000 y el enfoque a calidad del producto o servicio \\
\hline 2 & Postventa & Servicio que se brinda al cliente después de la adquisición del bien. \\
\hline 3 & Devolución & Acción de efecto de volver algo a su estado previo \\
\hline 4 & $7 / 24$ & Servicio siete días a 24 horas \\
\hline 5 & CRM & Gestión de relaciones con los clientes \\
\hline 6 & Logística & Conjunto de medio para llevar a cabo un fin determinado hacia el cliente \\
\hline 7 & Empaque & Conjunto de materiales en el cual se envuelve una cosa \\
\hline
\end{tabular}




\begin{tabular}{|l|l|l|}
\hline No. & \multicolumn{1}{|c|}{ Variable } & \multicolumn{1}{c|}{ Explicación } \\
\hline 8 & Clientes & Persona que utiliza o adquiere un servicio o producto en forma frecuente \\
\hline 9 & Despacho & Acción de despachar o enviar un producto o servicio \\
\hline 10 & WEB & Página de internet para dar a conocer la empresa \\
\hline 11 & Servicio & Prestar apoyo o asistencia a alguien por medio de diversos medios. \\
\hline 12 & Investigación de Mercados & Técnica para recolectar datos, e interpretarlos para tomar decisiones en relación al mercado. \\
\hline 13 & Precio & Dinero que está dispuesto a pagar el cliente por un producto o servicios \\
\hline 14 & Ofertas & Conjunto de bienes y servicios que se ofrece en el mercado \\
\hline 15 & Producto & Cosa producida en una operación de producción tangible o intangible. \\
\hline 16 & Pedidos & Acción de encargar algo \\
\hline 17 & Inquietud & Duda o interrogante \\
\hline 18 & Facturación & Extender una factura \\
\hline 19 & WhatsApp & Aplicación que permite enviar y recibir mensajes a través de teléfono móvil \\
\hline
\end{tabular}

Fuente: elaboración propia

La técnica empleada fue la encuesta, la cual se aplicó a 16 directivos de empresas químicas en Medellín, durante el periodo de 2017-2018, para lo cual se consolidó un cuestionario con 19 preguntas cerradas enfocadas al mercadeo y dos preguntas abiertas que pretendían determinar la prospectiva de estas empresas al 2030 en sus procesos de mercadeo y ventas (ver tabla 2). Para el análisis se empleó el MICMAC.

\section{RESULTADOS}

En las organizaciones de químicos en Medellín, se detecta que influye para mejorar el mercadeo de químicos en el futuro para una organización 4.0 en relación con el desarrollo sostenible seis elementos de las 19 variables estudiadas: certificación de calidad y amigable con el medio ambiente, logística, es decir transportar adecuadamente, en el tiempo con precio justo; tener un CRM actualizado que ayude a mejorar en el futuro el servicio de postventa y ofertar 24 horas - siete días a la semana.

Sin embargo, destacan que los más preocupante es la devolución de empaques o productos químicos. En contraposición, factores como analizar el cliente, uso de redes no son elementos que se preocupan los gerentes hacia el 2030 (ver figura 1). 
Direct influence /dependence map

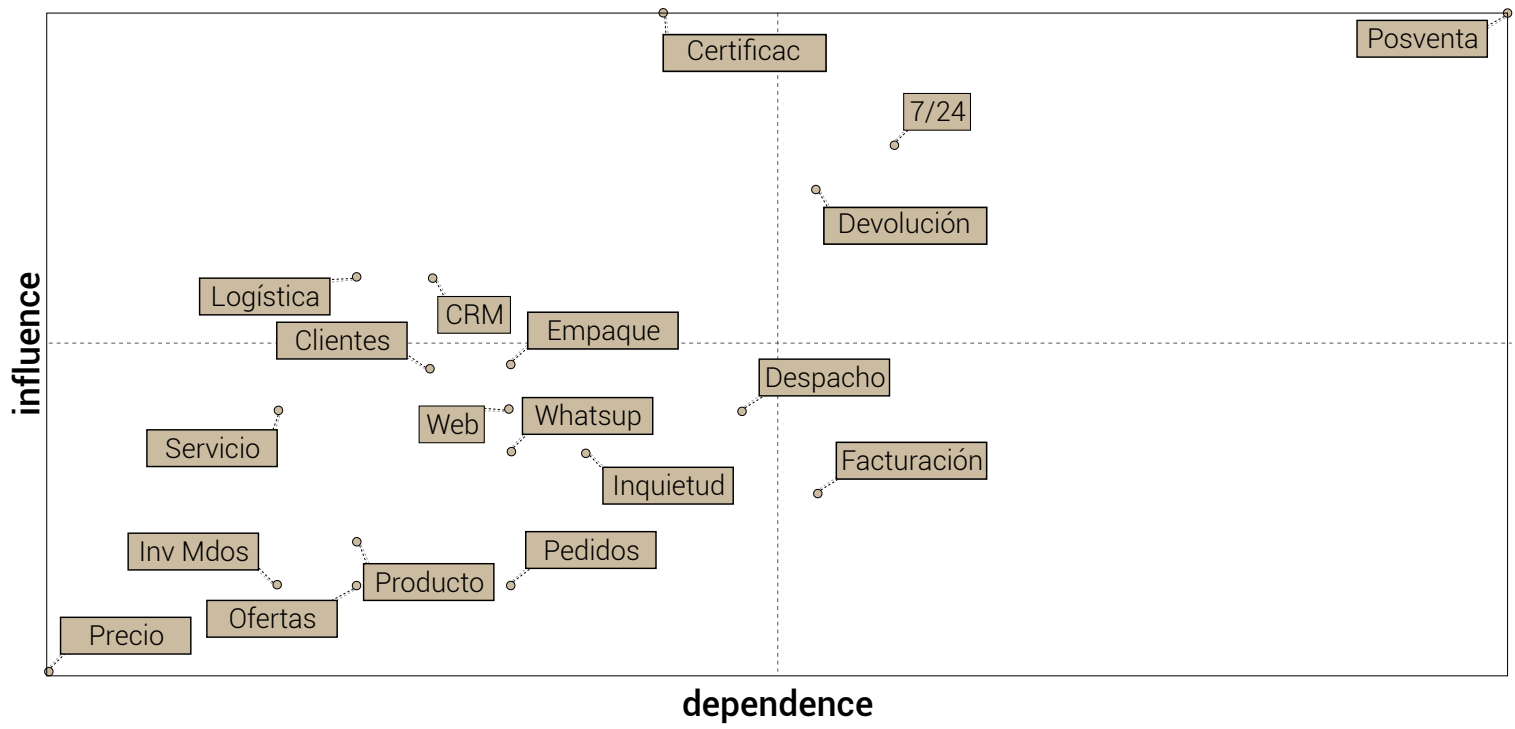

Figura 1. Influencia directa de empresas químicas.

Fuente: elaboración propia a partir del MICMAC (julio de 2019)

La figura precedente muestra la influencia directa que hay de 10 variables que afectan el desarrollo sostenible a futuro de las empresas químicas con una visión hacia el 2030 amparado en la innovación del mercadeo: productos, devolución, 7/24 horas de servicio, CRM, facturación electrónica, posventa productos amigables con el medio ambiente, servicio, ofertas, empaques y despacho oportuno en condiciones adecuadas. (ver figura 2).

Es decir, las organizaciones con un perfil de la 4,0 se deben preparar antes del 2030 en relación a comprender mejor el medioambiente y elaborar estrategias de mercadeo en relación a estas. En consecuencia, la cuarta revolución industria se enfoca hacia la hiperconectividad, después de haber pasado por la automatización de la empresa. En este caso, la industria de químicos debe enfocarse en ofrecer su mercancía en una forma diferente a la venta tradicional y apoyarse en la conectividad global por medio de intercambio de datos inteligentes con mayor información desde la automatización e reciprocidad de la tecnología. En otras palabras, mercadeo desde la sensórica, robótica, drones Big Data o por medio de la realidad aumentada. 


\section{Direct influence graph}

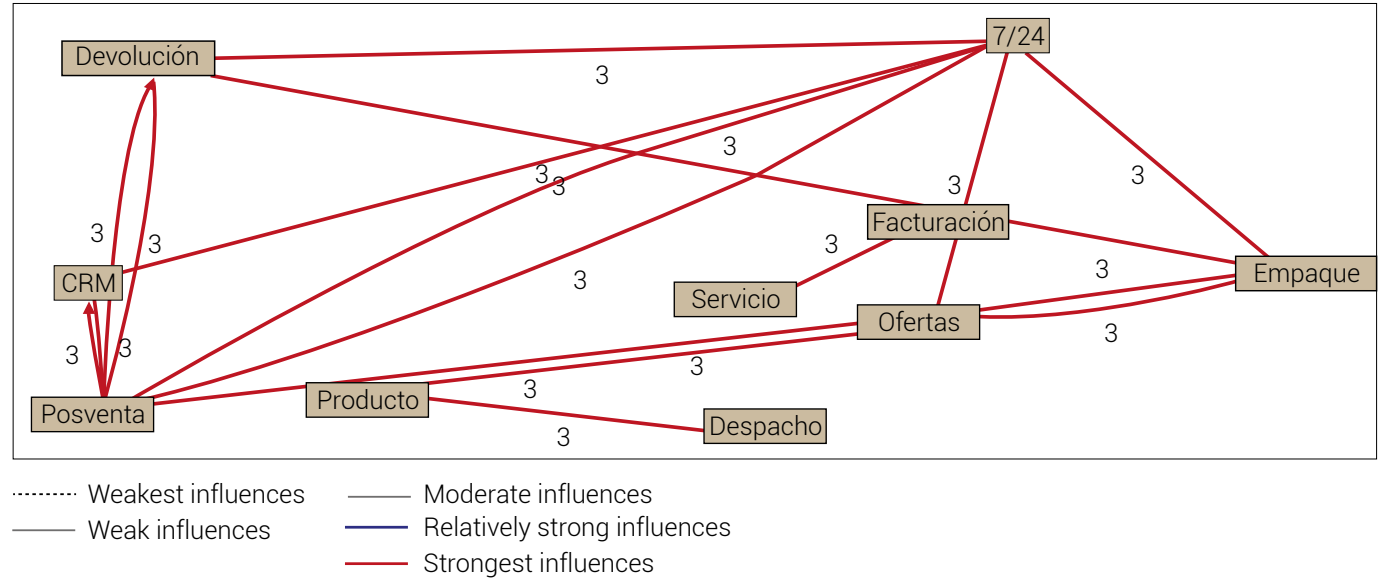

Figura 2. Influencia directa en empresas de químicos.

Fuente: elaboración propia a partir del MICMAC (julio de 2019)

Sin embargo, desde una visión indirecta de influencia de los elementos en estudio de empresa de químicos en Medellín se detecta que hacia el 2030 las empresas se deben centrar en otorgar certificaciones de calidad amigables, administrar el CRM, coordinar bien los procesos logísticos y servir con atención 7/24 horas apoyándose en prestar bien las estrategias de postventa. (ver figura 3).

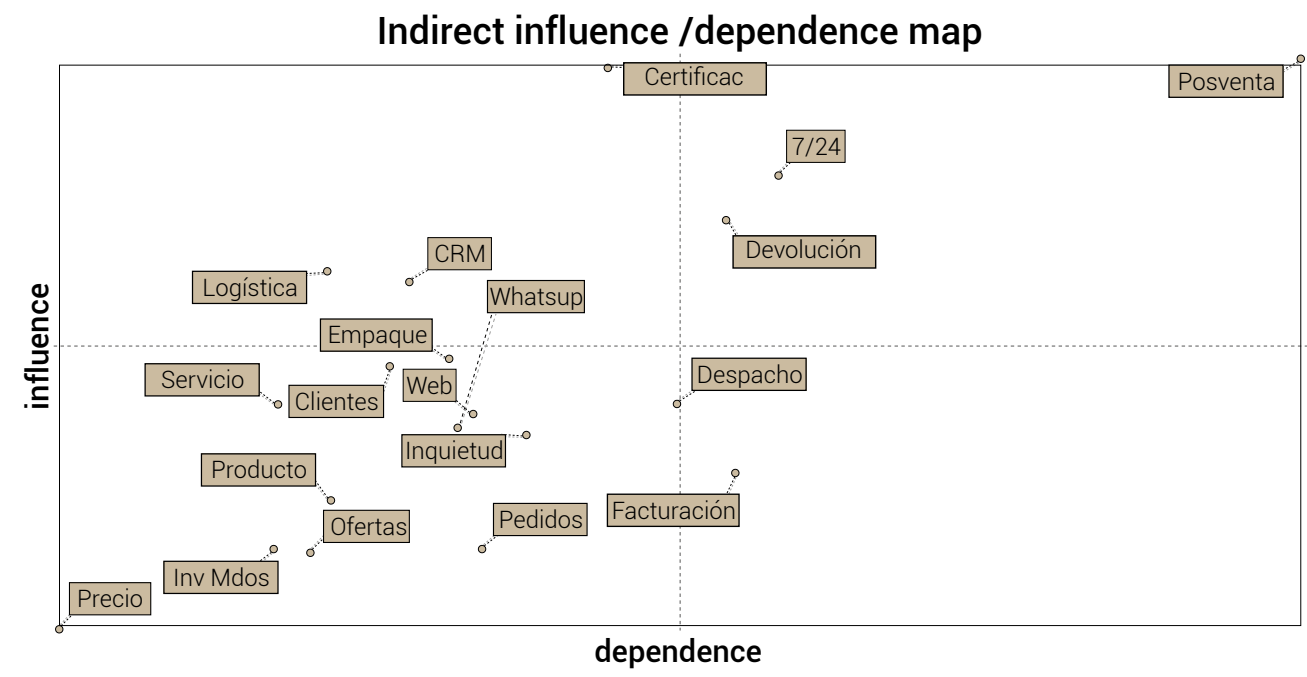

Figura 3. Influencia indirecta de empresas químicas.

Fuente: elaboración propia a partir del MICMAC (julio de 2019) 
Desde un análisis de influencia indirectas detecta que un factor esencial para las organizaciones futuras que incorporen las organizaciones 4,0 desde una visión de desarrollo sostenible es la certificación alineada con la gestión de postventa (ver figura 4).

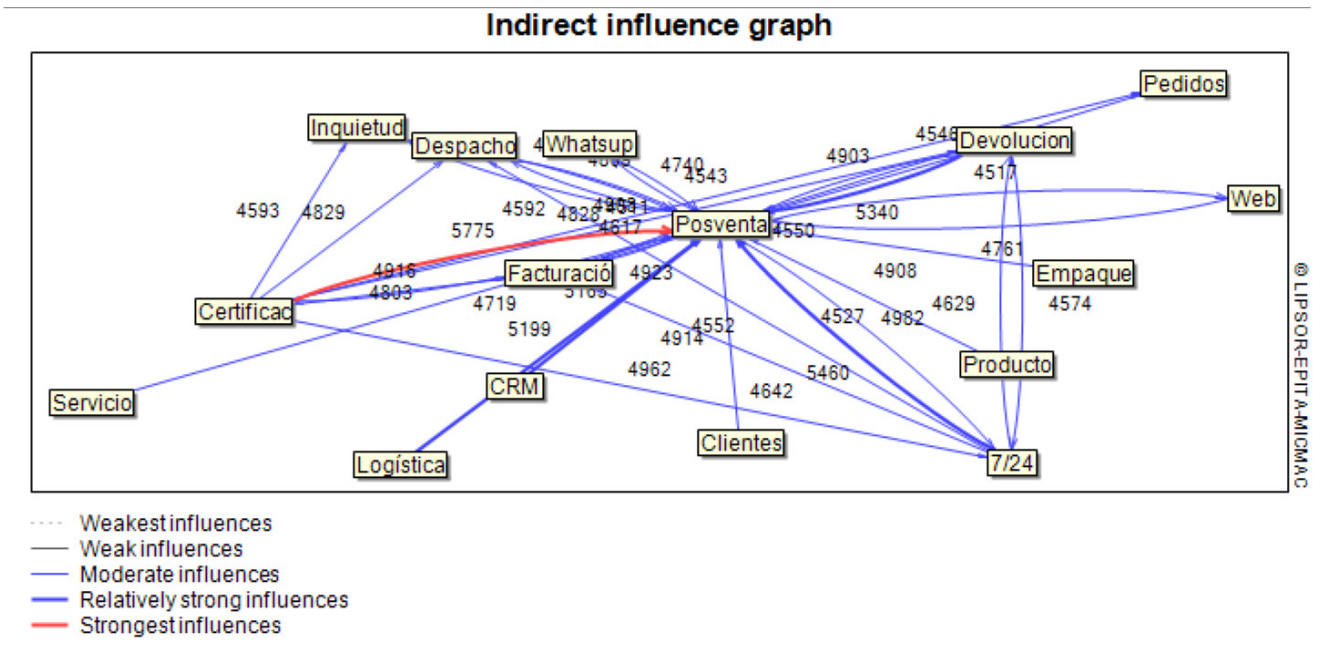

Figura 4. Influencia indirecta en empresas de químicos. Fuente: elaboración propia a partir del MICMAC (julio de 2019)

En síntesis, las organizaciones de químicos con un parámetro de ver sus empresas ligadas al desarrollo sostenible en el numeral 9 y con filosofía del vivir la 4,0 desde el mercadeo el factor indirecto es la certificación de calidad con postventa y de forma directa están certificación ambiental, CRM y logística.

\section{DISCUSIÓN}

A partir de los resultados, se puede destacar varios aspectos que permiten reconocer posiciones alternas, frente al valor que puede tener para una empresa del sector químico, como afrontar algunos retos; entre ellos, el conocer estándares de innovación; pero también, es el cómo se identifica como una empresa innovadora y además como implementa acciones para el desarrollo de la innovación. Esto permitirá registrar ¿cómo se configura la innovación en la empresa?, ¿qué lugar ocupa? ¿cuál es su utilidad? 
Unido a lo anterior se encuentran las devoluciones que pueden afectar el margen de ganancia; también de credibilidad en la empresa, así mismo el desarrollo de la confianza en el producto y la empresa. Adicional, esto puede generar malestar en la empresa al saber que el esfuerzo por un producto no ha permitido obtener el fin esperado. Es decir, aprender a utilizar los datos como medio para lograr un acercamiento al cliente desde el uso de la tecnología de la información Lasi, Fettke, Kemper, Feld, y Hoffmann (2014). En consecuencia, la realidad de las empresas a futuro debe enfocarse en el medio para conversar con el humano por medio de la tecnología (Brettel, Friederichsen, Keller, y Rosenberg, 2014), para impactar el sevicio venticuatro horas siete días a la semana. Todo esto, para tener información en tiempo real (Han, Lee y Choi, 2013) y coherente con lo que quiere el cliente y el comercializador (Mayer y Nusswald, 2001)

Un elemento adicional es el servicio 7/24, que genera un acercamiento al ser humano desde sus necesidades, intereses, posibilidades de esta en un contexto, el cual puede ser útil para forjar bienestar en el medio social y económico. Este servicio permite llegar a más personas, propiciar asesoría más amplia como también el ofertar servicios mayores. Posibilita que el cliente esté satisfecho con los productos químicos por su relación ambiental favorable.

Sin embargo, contradice lo expuesto por Karaosman, Morales-Alonso y Grijalvo (2015), en relación con definir bien la responsabilidad social empresarial desde un enfoque social y no ambiental; es decir, la conexión con los efectos de percepciones de la comunidad. Esto igualmente genera desde la visión empresarial retos como el disponer de personal cualificado, invertir en productos, servicios, entre otros. Así como el tener mayor personal que preste asesoría. Una asesoría que puede ser por la interacción del internet y la administración del Big Data (Ardito, Petruzzelli, Panniello y Garavelli, (2019) para evitar derrames de químicos o el cuidado que requiere el almacenamiento; pero sin olvidar la rentabilidad el bien ofertado Galletta, Carnevale, Celesti, Fazio e Illari (2017).

Esto, conjuntamente genera un conocimiento del producto. Necesariamente prioritario para ofertar un servicio 7/24 lo cual puede fomentar temor en la participación en este tipo de ejercicio. Lo antepuesto lleva también a analizar la facturación electrónica, como una acción que requiere tener recursos no solo físicos y digitales; es también tener recursos de conocimientos, el romper tradiciones, así mismo fragua transformaciones en el ámbito social, económico como también de 
posicionamiento del ser humano. Sin embargo, se propone tener un seguridad empresarial en los dispositivos para interactuar de forma segura con el cliente, como lo establece Ferrare (2019). En consecuencia, las empresa de químicos deben asegurar que la información de los clientes no este disponible en forma abierta. Además, se debe configurar un protocolo de seguridad en los nuevos sistemas que requieren las compañías.

La facturación electrónica afiliarse a proponer empalmes entre la tradición en algunos sectores, como también se visibiliza formas nuevas de competir en el mercado. Sin embargo, esta acción, algunos la consideran como poco pertinente para el desarrollo empresarial, debido a que sigue vigente la tradición del trato de la palabra como respaldo, de la contabilidad manual, el ajuste de prepuestos según las necesidades y el considerar las perdidas como ganancias y que simultáneamente son pasajeras. Pero, Wang et al., (2019), describe que se deben efectuar revisiones de los sietemas para garantizar las seguridad de los datos de los clientes, como es el caso de tener al día el CRM y el contacto continuo con el cliente.

Otro aspecto, es el evitar el señalamiento de personas y empresas de quienes no acceden a un pago; además, es una una forma de proteger el capital de las acciones de seguimiento digital que pueden ser sospechosos y desarrollar peligro para las inversiones como para las ganancias de la empresa; pero se puede evitar al configurar un protocolo de trasmisión de datos en tiempo real (Alani y Alloghani, 2019) afirnzando la seguridad de los datos de los clientes.

Un elemento de contingencia son los productos amigables con el medio ambiente en relación a este ítem; es decir, se visibiliza que los sectores empresariales cada vez buscan formas de estar atentos a este desarrollo y minimizar el impacto negativo en el medio ambiente, como está en el numeral 9 del desarrollo sostenible. En otras palabras, las TIC asumen un rol de integración de lo social, ambiental y financiero (Mrugalska y Wyrwicka, 2017) para que esto se transforme en valor desde dentro y externamente de la organización.

Sin embargo, se enfrenta a dos posiciones, una que considera que estos productos no son de la misma calidad de antes, que ha bajado su eficiencia y eficacia, por tanto, en expresiones de ellos no sirven; la segunda posición que estos productos al reinventarse se transforman, ya no se ofrece el original. De hecho, se detecta que los químicos en sí no afectan el medio 
ambiente si estos se gerencia de forma responsable. Pero hoy, se puede controlar mejor la gestión al vincular la tecnología en relación al control de seguridad al asimilar innovación que sea amigable con el medio ambiente (Vaidya, Ambad, y Bhosle, 2018).

Así, se es amigable con el medio ambiente, empero, se corre el riego de tener menos posibilidades de sostener los clientes, por ello, se debe salir a buscar clientes nuevos que por lo general son jóvenes. El contacto con la experiencia y lo novel a veces funciona, pero en otras no, porque estas poblaciones tienen diferentes visiones empresariales con nuevas tecnologías aportadas de la organización 4.0 para lograr un equilibrio de transacción de calidad, servicio y gestión para lograr mayor compromiso de mercadeo por parte de las organizaciones comercializadoras de químicos, como lo expone Sony y Naik (2019),

En relación a las Ofertas, esta acción crea posibilidades no solo de atraer a los clientes habituales y nuevos, es también de pensarse en como favorecer a los diversos grupos que atiende. Permite un desarrollo de nuevos mercados. Lo cual va unido entre otros a los empaques, aunque representa la imagen de la empresa, son una forma de tener cercanía con la comunidad y determinan posicionamiento en un grupo, pero son medios de contacto. Es decir, asegurar un empaque que promueva el posicionamiento de marca y de la calidad del producto en relación con el medio ambiente (Roblek, Meško y Krapež, 2016).

Por ello el despacho oportuno en condiciones adecuadas significa oportunidad de satisfacer el mercado, retener clientes y generar respeto. Así mismo, consolida una posición en el mercado. Ayuda a brindar seguridad en que el producto está adecuado, en las mejores condiciones. Dispensa para la empresa, credibilidad y disminuye el margen de perdida. También es la posibilidad de establecer margen de competencia entre empresas por el servicio que ofertan.

\section{CONCLUSIONES}

Las empresas químicas en Medellín, cuando se reconocen como una organización 4.0 o que al menos están transitando hacia ello, deben atender transformaciones históricas tanto para el sector como de tipo de formación, de quienes las crearon como también de una sociedad que todavía media entre la tradición. Adicional, avizorar una organización con una directa vivencia de 4.0, todavía en este sector químico, debe atender a una reconstrucción de los procesos de 
alineación tanto de los futuros integrantes de estas empresas como también de quienes ya ejercen su labor directiva.

Así mismo, es mediar en una cultura donde todavía el saber ancestral prima sobre el tecnológico y este va unido al respaldo del ser en sociedad donde la persona se consolida como eje central de toda relación, por ello, se les dificulta el integrar algunos aspectos que conlleva organizaciones 4.0. Es una interacción entre la tradición y los nuevos espacios de transformación del sector financiero.

Quizá, este sentido de nueva forma de organizar la empresa, sus atributos, pueda desarrollar mejoras en todas las áreas empresariales, pero es necesario hacer un paso orientado, consensuado, escalonado y en especial consciente por parte de los seres que habitan la empresa y de los clientes.

En síntesis, cuando una empresa se orienta a atender las demandas que puede generar los procesos de certificación, logística y gestión de las relaciones del consumidos (CMR), con el fin de mejorar su interacción con el cliente y fortalecer el desarrollo sostenible.

\section{REFERENCIAS}

Alani, M. M., and Alloghani, M. (2019). Security Challenges in the Industry 4.0 Era. In Industry 4.0 and Engineering for a Sustainable Future (pp. 117-136). Springer.

Ardito, L., Petruzzelli, A. M., Panniello, U., and Garavelli, A. C. (2019). Towards Industry 4.0: Mapping digital technologies for supply chain management-marketing integration. Business Process Management Journal, 25(2), 323-346.

Barreto, L., Amaral, A., and Pereira, T. (2017). Industry 4.0 implications in logistics: an overview. Procedia Manufacturing, 13, 1245-1252.

Brettel, M., Friederichsen, N., Keller, M., and Rosenberg, M. (2014). How virtualization, decentralization and network building change the manufacturing landscape: An Industry 4.0 Perspective. International Journal of Mechanical, Industrial Science and Engineering, 8(1), 37-44. 
Chikez, H. B. (2016). Establishing cycle times in a high variety-manufacturing environment using DMAIC Procedure (Doctoral dissertation, Stellenbosch: Stellenbosch University).

Ferrare, J. J. (2019). A Multi-Institutional Analysis of Instructional Beliefs and Practices in Gateway Courses to the Sciences. CBE-Life Sciences Education, 18(2), 26.

Fremont, V. H. J., Frick, J. E., Åge, L. J., and Osarenkhoe, A. (2019). Interaction through boundary objects: controversy and friction within digitalization. Marketing Intelligence y Planning, 37(1), 111-124.

Galletta, A., Carnevale, L., Celesti, A., Fazio, M., and Villari, M. (2017). A cloud-based system for improving retention marketing loyalty programs in industry 4.0 : a study on big data storage implications. IEEE Access, 6, 5485-5492.

Gornostaeva, Z. V., Lazareva, N. V., Bugaeva, M. V., Gribova, O. V., and Zibrova, N. M. (2018). Directions and tools of industry marketization in contemporary Russia. Quality-Access to Success, 19.

Han, K. H., Lee, G., and Choi, S. H. (2013). Manufacturing cycle time reduction for batch production in a shared worker environment. International Journal of Production Research, 51(1), 1-8.

Hernández Sampieri, R., Fernández Collado, C. y Baptista Lucio, P. (2010). Metodología de la investigación. Mc Graw Hill. México.

Henning, M., Hagedorn-Hansen, D., and Von Leipzig, K. H. (2018). A conceptual framework to increase competitiveness in a biltong factory. South African Journal of Industrial Engineering, 29(3), 108-120.

Kagermann, H., Helbig, J., Hellinger, A., and Wahlster, W. (2013). Recommendations for implementing the strategic initiative INDUSTRIE 4.0: Securing the future of German manufacturing industry; final report of the Industrie 4.0 Working Group. Forschungsunion.

Karaosman, H., Morales-Alonso, G., and Grijalvo, M. (2015). Consumers' responses to CSR in a cross-cultural setting. Cogent Business y Management, 2(1), 1052916. 
Lasi, H., Fettke, P., Kemper, H.-G., Feld, T., and Hoffmann, M. (2014). Industry 4.0. Business y Information Systems Engineering, 6(4), 239-242.

Li, L. (2018). China's manufacturing locus in 2025: With a comparison of "Made-in-China 2025" and "Industry 4.0". Technological Forecasting and Social Change, 135, 66-74.

Mrugalska, B., and Wyrwicka, M. K. (2017). Towards lean production in industry 4.0. Procedia Engineering, $182,466-473$.

Mayer, M., and Nusswald, M. (2001). Improving manufacturing costs and lead times with quality-oriented operating curves. Journal of Materials Processing Technology, 119(1-3), 83-89.

Nadarajah, S., and Kotz, S. (2008). The cycle time distribution. International Journal of Production Research, 46, (11), 3133-3141.

ONU (2015). Objetivos de desarrollo sostenible. Recuperado de https://www.un.org/sustainabledevelopment/es/infrastructure/

Palazzeschi, L., Bucci, O., and Di Fabio, A. (2018). Re-thinking innovation in organizations in the industry 4.0 scenario: New challenges in a primary prevention perspective. Frontiers in Psychology, 9, 30.

Pereira, A. C., and Romero, F. (2017). A review of the meanings and the implications of the Industry 4.0 concept. Procedia Manufacturing, 13, 1206-1214.

Roblek, V., Meško, M., and Krapež, A. (2016). A complex view of industry 4.0. Sage Open, 6(2).

Shipton, H. J., West, M. A., Parkes, C. L., Dawson, J. F., and Patterson, M. G. (2006). When promoting positive feelings pays: Aggregate job satisfaction, work design features, and innovation in manufacturing organizations. European Journal of Work and Organizational Psychology, 15(4), 404-430. 
Sony, M., and Naik, S. (2019). Key ingredients for evaluating Industry 4.0 readiness for organizations: a literature review. Benchmarking: An International Journal.

Vaidya, S., Ambad, P., and Bhosle, S. (2018). Industry 4.0-a glimpse. Procedia Manufacturing, 20, 233-238.

Wang, B., Wu, L., Li, D., Liu, Y., Guo, J., Li, C., and Fu, M. (2019). Induction of Pluripotency by Alternative Factors. 